\title{
Article
}

\section{A Note on a Triple Integral}

\author{
Robert Reynolds*(i) and Allan Stauffer (i)
}

Department of Mathematics and Statistics, York University, Toronto, ON M3J 1P3, Canada; stauffer@yorku.ca

* Correspondence: milver@my.yorku.ca

Abstract: A closed form expression for a triple integral not previously considered is derived, in terms of the Lerch function. Almost all Lerch functions have an asymmetrical zero-distribution. The kernel of the integral involves the product of the logarithmic, exponential, quotient radical, and polynomial functions. Special cases are derived in terms of fundamental constants; results are summarized in a table. All results in this work are new.

Keywords: triple integral; Lerch function; Catalan's constant; Apéry's constant; Euler's constant; Glaisher's constant; Cauchy integral formula

MSC: Primary 30E20; 33-01; 33-03; 33-04; 33-33B; 33E20

check for updates

Citation: Reynolds, R.; Stauffer, A. A Note on a Triple Integral. Symmetry 2021, 13, 2056. https://doi.org/ 10.3390/sym 13112056

Academic Editors: Marin Marin, Abdelmejid Bayad and Yilmaz Simsek

Received: 26 September 2021

Accepted: 28 October 2021

Published: 1 November 2021

Publisher's Note: MDPI stays neutral with regard to jurisdictional claims in published maps and institutional affiliations.

Copyright: (c) 2021 by the authors. Licensee MDPI, Basel, Switzerland. This article is an open access article distributed under the terms and conditions of the Creative Commons Attribution (CC BY) license (https:// creativecommons.org/licenses/by/ $4.0 /)$.

\section{Significance Statement}

Triple integrals are used in many areas of mathematics and physics. Some useful applications of these integrals are in finding the the mass of a ball of radius, say (r) whose density $(\rho)$ is proportional to the squared distance from the center; another application is in finding the moment of inertia of a right circular homogeneous cone about its axis.

These integrals are also used to find the mass of a planet, where its radius and density are expressed in terms of a formula. Triple integrals are also used in calculating the volume of a region of a space within a given region in the metric of Euclidean space [1], calculating the conditional probability density function in Bayesian methods used in cosmology [2], the evaluation of cosmic strings in the weak-field approximation [3] and in the calculation of the astrophysical term in the study of Cosmic Rays for Particle and Astroparticle Physics [4].

Based on our short reading of current literature involving triple integrals, there seems to be a lack of closed form solutions for the given triple integrals in a general form. Our present work aims to supply a formal derivation and a specific triple integral, and express it in terms of a special function. These results will provide a new process and formula for current work requiring such results.

\section{Introduction}

In this work, a general integral theorem was developed, from which integral and closed form results, in terms of the Lerch function, Hurwitz zeta function, polylogarithm function, and the Riemann zeta function could be expressed. The definite integral obtained in this present work is given by

$$
\int_{0}^{\infty} \int_{0}^{\infty} \int_{0}^{\infty} z^{-\frac{m n}{2}-1}(x y)^{m / 2}(x+y)^{-\frac{m}{2}-\frac{1}{2}} \log ^{k}\left(\frac{a z^{-n / 2} \sqrt{x y}}{\sqrt{x+y}}\right) e^{-\beta z^{n}-p x-q y} d x d y d z
$$

where the parameters $k, a$ are general complex numbers and $\operatorname{Re}(\beta)>0, \operatorname{Re}(p)>0, \operatorname{Re}(q)>0$, $\operatorname{Re}(m) \leq 0$. This integral with integer values of the parameters yield expressions in terms of fundamental constants namely Catalan's constant $C$, equation (25.11.40) in [5], Glaisher's constant, equation (5.17.6) in [5], Apéry's constant, equation (25.6.9) in [5] and Euler's constant, equation (5.2.3) in [5]. 
The definite integral was obtained using the method described in [6], which includes Cauchy's integral formula. The generalized Cauchy's integral formula is given by

$$
\frac{y^{k}}{\Gamma(k+1)}=\frac{1}{2 \pi i} \int_{\Delta} \frac{e^{w y}}{w^{k+1}} d w .
$$

where $\Delta$ is, in general, an open contour in the complex plane where the bilinear concomitant has the same value at the end points of the contour. This method includes multiplying both sides of (2) by a function, then taking a definite triple integral of both sides by using a form of Equation (2). In terms of a contour integral, this produces a triple definite integral. A second contour integral was obtained by multiplying Equation (2) by a function, making certain substitutions, then taking the infinite sum to obtain the same contour integrals.

\section{Definite Integral of the Contour Integral}

The variable of integration of the contour integral is $t=m+w$. The cut and contour are in the first quadrant of the complex $t$-plane described in [6]. We use Equation (2) and replace $y$ by $\log \left(\frac{a z^{-n / 2} \sqrt{x y}}{\sqrt{x+y}}\right)$ then multiply by $z^{-\frac{m n}{2}-1}(x y)^{m / 2}(x+y)^{-\frac{m}{2}-\frac{1}{2}} e^{-\beta z^{n}-p x-q y}$. Next, we take the triple infinite integral over $x \in[0, \infty), y \in[0, \infty)$ and $z \in[0, \infty)$ to get

$$
\begin{aligned}
& \frac{1}{\Gamma(k+1)} \int_{0}^{\infty} \int_{0}^{\infty} \int_{0}^{\infty} z^{-\frac{m n}{2}-1}(x y)^{m / 2}(x+y)^{-\frac{m}{2}-\frac{1}{2}} \log ^{k}\left(\frac{a z^{-n / 2} \sqrt{x y}}{\sqrt{x+y}}\right) e^{-\beta z^{n}-p x-q y} d x d y d z \\
& =\frac{1}{2 \pi i} \int_{0}^{\infty} \int_{0}^{\infty} \int_{0}^{\infty} \int_{\Delta} a^{w} w^{-k-1} z^{-\frac{1}{2} n(m+w)-1}(x y)^{\frac{m+w}{2}}(x+y)^{\frac{1}{2}(-m-w)-\frac{1}{2}} e^{-\beta z^{n}-p x-q y} d w d x d y d z \\
& =\frac{1}{2 \pi i} \int_{\Delta} \int_{0}^{\infty} \int_{0}^{\infty} \int_{0}^{\infty} a^{w} w^{-k-1} z^{-\frac{1}{2} n(m+w)-1}(x y)^{\frac{m+w}{2}}(x+y)^{\frac{1}{2}(-m-w)-\frac{1}{2}} \\
& e^{-\beta z^{n}-p x-q y} d x d y d z d w \\
& =-\frac{1}{2 \pi i} \int_{\Delta} \frac{\pi^{3 / 2} a^{w} w^{-k-1} \beta^{\frac{m+w}{2}} \csc \left(\frac{1}{2} \pi(m+w)\right)(\sqrt{p}+\sqrt{q})^{-m-w-1}}{n \sqrt{p q}} d w
\end{aligned}
$$

from equations (3.326.2) in [7] and (3.1.3.9) in [8] where $\operatorname{Re}(w+m)>0, \operatorname{Re}(\beta)>0, \operatorname{Re}(p)>$ $0, \operatorname{Re}(q)>0, \operatorname{Re}(m) \leq 0$ and using the reflection formula for the Gamma function in equation (8.334.2) in [7]. We can reverse the order of integration over $t, x y$ and $z$ using Fubini's theorem due to the integrand being of bounded measure over the space $C \times$ $[0, \infty) \times[0, \infty) \times[0, \infty)$, where $\Delta$ is the non-zero complex plane.

\section{The Lerch Function}

We use equation (1.11.3) in [9-11] where $\Phi(z, s, v)$ is the Lerch function, which is a generalization of the Hurwitz zeta $\zeta(s, v)$ and polylogarithm functions $L i_{n}(z)$. The Lerch function has a series representation given by

$$
\Phi(z, s, v)=\sum_{n=0}^{\infty}(v+n)^{-s} z^{n}
$$

where $|z|<1, v \neq 0,-1,-2,-3, .$. , and is continued analytically by its integral representation given by

$$
\Phi(z, s, v)=\frac{1}{\Gamma(s)} \int_{0}^{\infty} \frac{t^{s-1} e^{-(v-1) t}}{e^{t}-z} d t
$$

where $\operatorname{Re}(v)>0$, and either $|z| \leq 1, z \neq 1, \operatorname{Re}(s)>0$, or $z=1, \operatorname{Re}(s)>1$.

\section{Infinite Sum of the Contour Integral}

Again, using Cauchy's integral Formula (2), and taking the infinite sum to derive an equivalent sum representation for the contour integral, we proceed using Equation (2) and replace $y$ by $\log (a)+\frac{\log (\beta)}{2}-\log (\sqrt{p}+\sqrt{q})+\frac{1}{2} i \pi(2 y+1)$, and multiply both sides 
by $\frac{2 i \pi^{3 / 2} \beta^{m / 2}(\sqrt{p}+\sqrt{q})^{-m-1}}{n \sqrt{p q}}$, then taking the infinite sum over $y \in[0, \infty)$, and simplifying in terms of the Lerch function to get

$$
\begin{aligned}
& \frac{1}{n \Gamma(k+1) \sqrt{p q}} 2 i \pi^{k+\frac{3}{2}} e^{\frac{1}{2} i \pi(k+m)} \beta^{m / 2}(\sqrt{p}+\sqrt{q})^{-m-1} \\
& \Phi\left(e^{i m \pi},-k,-\frac{i(2 \log (a)-2 \log (\sqrt{p}+\sqrt{q})+\log (\beta)+i \pi)}{2 \pi}\right) \\
& =\frac{1}{2 \pi i} \sum_{y=0}^{\infty} \int_{\Delta} \frac{2 i \pi^{3 / 2} a^{w} w^{-k-1} \beta^{\frac{m+w}{2}} e^{\frac{1}{2} i \pi(2 y+1)(m+w)}(\sqrt{p}+\sqrt{q})^{-m-w-1}}{n \sqrt{p q}} d w \\
& =\frac{1}{2 \pi i} \int_{\Delta} \sum_{y=0}^{\infty} \frac{2 i \pi^{3 / 2} a^{w} w^{-k-1} \beta^{\frac{m+w}{2}} e^{\frac{1}{2} i \pi(2 y+1)(m+w)}(\sqrt{p}+\sqrt{q})^{-m-w-1}}{n \sqrt{p q}} d w \\
& =-\frac{1}{2 \pi i} \int_{\Delta} \frac{\pi^{3 / 2} a^{w} w^{-k-1} \beta^{\frac{m+w}{2}} \csc \left(\frac{1}{2} \pi(m+w)\right)(\sqrt{p}+\sqrt{q})^{-m-w-1}}{n \sqrt{p q}} d w
\end{aligned}
$$

from Equation (1.232.3) in [7] where $\operatorname{Im}(w+m)>0$ in order for the sum to converge.

Theorem 1. For $k, a \in \mathbb{C}, \operatorname{Re}(p)>0, \operatorname{Re}(q)>0, \operatorname{Re}(n)>0, \operatorname{Re}(\beta)>0, \operatorname{Re}(m) \leq 0$,

$$
\begin{aligned}
& \int_{0}^{\infty} \int_{0}^{\infty} \int_{0}^{\infty} z^{-\frac{m n}{2}-1}(x y)^{m / 2}(x+y)^{-\frac{m}{2}-\frac{1}{2}} \log ^{k}\left(\frac{a z^{-n / 2} \sqrt{x y}}{\sqrt{x+y}}\right) e^{-\beta z^{n}-p x-q y} d x d y d z \\
& =\frac{1}{n \sqrt{p q}} 2 i \pi^{k+\frac{3}{2}} e^{\frac{1}{2} i \pi(k+m)} \beta^{m / 2}(\sqrt{p}+\sqrt{q})^{-m-1} \\
& \Phi\left(e^{i m \pi},-k,-\frac{i(2 \log (a)-2 \log (\sqrt{p}+\sqrt{q})+\log (\beta)+i \pi)}{2 \pi}\right)
\end{aligned}
$$

Proof. The right-hand sides of relations (3) and (6) are identical; hence, the left-hand sides of the same are identical too. Simplifying with the Gamma function yields the desired conclusion.

\section{Derivation of Table Entries}

All constants in the Table 1 of Integrals are obtainable from relation (7); hence, by an evaluation of a triple integral. The constants evaluated in the following Table 1 are three-dimensional in nature. This may have important cosmological consequences yet to be discovered by further studies.

Entry 1

Catalan's constant $C$. Use Equation (7) and set $k=-2, m=-1, a=-1, \beta=1$, $p=q=1 / 4, n=3$, rationalize the denominator equate real and imaginary parts, and simplify using entry (4) in the Table 1 below (64:12:7) and equation (64:4:1) in [12] and equation (2.2.1.2.7) in [13].

Entry 2

Glaisher's constant $A$. Use Equation (7) and set $k=1, m=-1, a=i, \beta=1, p=q=1 / 4$, $n=2$, and simplify using equation (8) in [14].

Entry 3

Apéry's constant $\zeta(3)$. Use Equation (7), take the first partial derivative with respect to $k$ and set $k=2, m=-1, a=i, \beta=1, p=q=1 / 4, n=1$, and simplify using Equation (9) in [14].

Entry 4

Use Equation (7) and set $m=-1, \beta=1, p=q=1 / 4, n=1, a=-1, n=4$ and apply l'Hopital's rule as $k \rightarrow-1$, simplify using entry (4) in the table below (64:12:7) and equation (64:9:2) in [12].

\section{Entry 5}

Riemann zeta function $\zeta(k)$. Use Equation (7) and set $m=-1, \beta=1, p=q=1 / 4$, $n=1, a=i, n=4$, and simplify using entry (4) in the table below (64:12:7) and entry (2) in the table below (64:7) in [12]. 


\section{Entry 6}

Apéry's constant $\zeta(3)$. Use entry (5) and set $k=-3, n=8$ and simplify. Entry 7

$\zeta(5)$. Use entry (5) and set $k=-5, n=8$ and simplify.

Entry 8

Use Equation (7) and obtain a second equation by replacing $m \rightarrow r$, and taking the difference. Next, set $k=-1, a=1, \beta=1, p=q=1 / 4, m=-2 / 3, r=-3 / 4$ and simplify using entry (1) in the table below (64:12:7) in [12].

Entry 9

Use Equation (7) and obtain a second equation by replacing $m \rightarrow r$ and taking the difference. Next, set $k=-1, a=1, \beta=1, p=q=1 / 4, m=-4 / 5, r=-5 / 6$ and simplify using entry (1) in the table below (64:12:7) in [12].

Entry 10

Use Equation (7) and obtain a second equation by replacing $m \rightarrow r$ and taking the difference. Next, set $k=-1, a=1, \beta=1, p=q=1 / 4, m=-1, r=-1 / 2$ and simplify using entry (1) in the table below (64:12:7) in [12].

Entry 11

Use Equation (7) and obtain a second equation by replacing $m \rightarrow r$ and taking the difference. Next set $k=-1, a=1, \beta=1, p=q=1 / 4, m=-1 / 4, r=-1 / 2$ and simplify using entry (1) in the table below (64:12:7) in [12].

Entry 12

Euler's constant $\gamma$. Use entry (5) apply l'Hopital's rule as $k \rightarrow-1$ and simplify using Equation (5.17.7) in [5].

Table 1. Table of Integrals.

\begin{tabular}{|c|c|}
\hline$f(x, y, z)$ & $\int_{0}^{\infty} \int_{0}^{\infty} \int_{0}^{\infty} f(x, y, z) d x d y d z$ \\
\hline 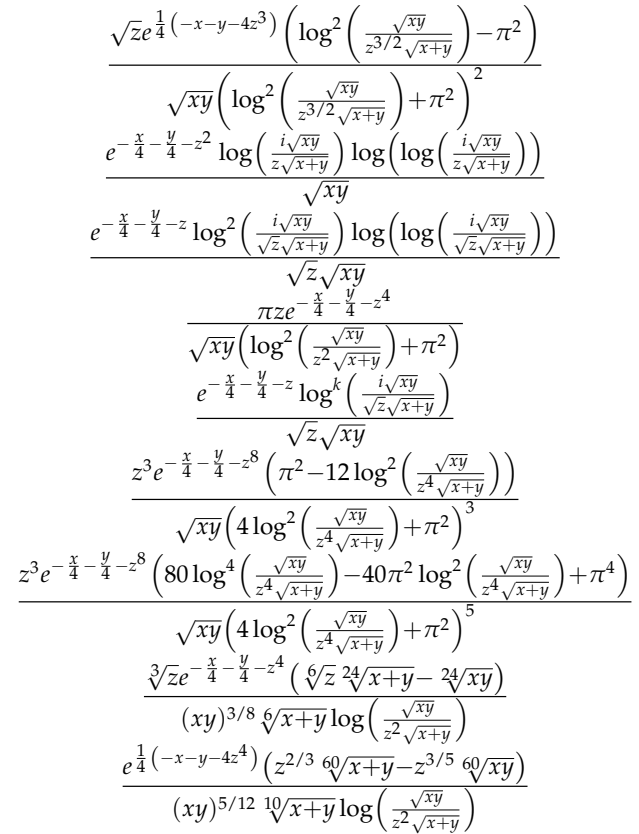 & $\begin{array}{c}\frac{32(C-1)}{3 \sqrt{\pi}} \\
-4 i \pi^{5 / 2} \log \left(\frac{A^{3}}{\sqrt[3]{2} \sqrt[4]{e}}\right)-\frac{\pi^{7 / 2}}{2}+i \pi^{5 / 2} \log (\pi) \\
14 \pi^{3 / 2} \zeta(3) \\
(4-\pi) \sqrt{\pi} \\
-8\left(2^{k+1}-1\right) e^{\frac{i \pi k}{2}} \pi^{k+\frac{3}{2}} \zeta(-k) \\
\frac{3 \zeta(3)}{32 \pi^{5 / 2}} \\
\frac{15 \zeta(5)}{512 \pi^{9 / 2}} \\
\sqrt{\pi} \log \left(\frac{2}{3-3 \sin \left(\frac{\pi}{8}\right)}-\frac{1}{3}\right) \\
\sqrt{\pi} \log \left(\frac{(\sqrt{5}-5)(4-\sqrt{2}+\sqrt{6})}{(3+\sqrt{5})(-4-\sqrt{2}+\sqrt{6})}\right)\end{array}$ \\
\hline
\end{tabular}


Table 1. Cont.

\begin{tabular}{|c|c|}
\hline$f(x, y, z)$ & $\int_{0}^{\infty} \int_{0}^{\infty} \int_{0}^{\infty} f(x, y, z) d x d y d z$ \\
\hline$e^{-\frac{x}{4}-\frac{y}{4}-z^{4}}(\sqrt[4]{x y}-z \sqrt[4]{x+y})$ & \multirow{2}{*}{$2 \sqrt{\pi} \operatorname{coth}^{-1}(\sqrt{2})$} \\
\hline$\sqrt{x y} \sqrt[4]{x+y} \log \left(\frac{\sqrt{x y}}{z^{2} \sqrt{x+y}}\right)$ & \\
\hline$e^{-\frac{x}{4}-\frac{y}{4}-z^{4}}(\sqrt{z} \sqrt[8]{x+y}-\sqrt[8]{x y})$ & \multirow{2}{*}{$\sqrt{\pi}\left(\cosh ^{-1}(3)+2 \log \left(\tan \left(\frac{\pi}{16}\right)\right)\right)$} \\
\hline$\sqrt{z} \sqrt[4]{x y}(x+y)^{3 / 8} \log \left(\frac{\sqrt{x y}}{z^{2} \sqrt{x+y}}\right)$ & \\
\hline$e^{-\frac{x}{4}-\frac{y}{4}-z} \log \left(\log \left(\frac{i \sqrt{x y}}{\sqrt{z} \sqrt{x+y}}\right)\right)$ & \multirow[t]{2}{*}{$4 \sqrt{\pi} \log (2)\left(2 i \gamma+\pi-i \log \left(2 \pi^{2}\right)\right)$} \\
\hline$\sqrt{z} \sqrt{x y} \log \left(\frac{i \sqrt{x y}}{\sqrt{z} \sqrt{x+y}}\right)$ & \\
\hline
\end{tabular}

\section{An Example Using Non-Integer Values}

Using Equation (7) and set $k=-2 i, a=-1 / 3, p=\pi, q=2 \pi, n=5 / 2, \beta=5 / 3$, $m=-5 / 6$.

$$
\begin{aligned}
& \int_{0}^{\infty} \int_{0}^{\infty} \int_{0}^{\infty} \frac{1}{(x y)^{5 / 12}} \sqrt[12]{x+y} \sqrt[24]{z} e^{-\pi(x+2 y)-\frac{5 z^{5 / 2}}{3}} \\
& \log { }^{-2 i}\left(-\frac{\sqrt{x y}}{3 z^{5 / 4} \sqrt{x+y}}\right) d x d y d z \\
& =-\frac{2}{5} i(-1)^{\frac{7}{12}-i}\left(\frac{3}{5}\right)^{5 / 12} \sqrt{2} \sqrt[6]{\sqrt{2}-1} \pi \frac{5}{12}-2 i \\
& \Phi\left(-\sqrt[6]{-1}, 2 i, \frac{3}{2}+\frac{i\left(2 \sinh ^{-1}(1)+\log \left(\frac{27 \pi}{5}\right)\right)}{2 \pi}\right)
\end{aligned}
$$

\section{Discussion}

Using our contour integration method, we offered a novel method for generating several intriguing triple integrals in this study. We demonstrated the versatility of our method by merging two different definite integrals to generate a triple integral. Using our method, we will continue our research of these types of integrals and expand the summary Table 1 . The results were mathematically validated using Wolfram Mathematica for both real and imaginary and complex values of the parameters in the integrals.

Author Contributions: Conceptualization, R.R., supervision, A.S., funding acquisition, A.S. All authors have read and agreed to the published version of the manuscript.

Funding: This research is supported by NSERC Canada under grant 504070.

Conflicts of Interest: The authors declare no conflict of interest.

\section{References}

1. Lachiéze-Rey, M. Cosmology: A First Course; Cambridge University Press: Cambridge, UK, 1995.

2. Hobson, M.P.; Jaffe, A.H.; Liddle, A.R. Bayesian Methods in Cosmology; Cambridge University Press: Cambridge, UK, 2010.

3. Anderson, M.R. The Mathematical Theory of Cosmic Strings Cosmic Strings in the Wire Approximation; CRC Press: Boca Raton, FL, USA, 2015.

4. Leroy, C.; Rancoita, P.-G.; Giani, S. Cosmic Rays for Particle and Astroparticle Physics. In Proceedings of the 12th ICATPP Conference, Villa Olmo, Como, Italy, 7-8 October 2010.

5. Olver, F.W.J.; Lozier, D.W.; Boisvert, R.F.; Clark, C.W. (Eds.) NIST Digital Library of Mathematical Functions; U.S. Department of Commerce, National Institute of Standards and Technology: Washington, DC, USA; Cambridge University Press: Cambridge, UK, 2010; With 1 CD-ROM (Windows, Macintosh and UNIX). MR 2723248 (2012a:33001).

6. Reynolds, R.; Stauffer, A. A Method for Evaluating Definite Integrals in Terms of Special Functions with Examples. Int. Math. Forum 2020, 15, 235-244. [CrossRef]

7. Gradshteyn, I.S.; Ryzhik, I.M. Tables of Integrals, Series and Products, 6th ed.; Academic Press: Cambridge, MA, USA, 2000.

8. Prudnikov, A.P.; Brychkov, Y.A.; Marichev, O.I. Integrals and Series, More Special Functions; USSR Academy of Sciences: Moscow, Russia, 1990; Volume 1.

9. Erdéyli, A.; Magnus, W.; Oberhettinger, F.; Tricomi, F.G. Higher Transcendental Functions; McGraw-Hill Book Company, Inc.: New York, NY, USA; Toronto, ON, Canada; London, UK, 1953; Volume I.

10. Srivastava, H.M. Some general families of the Hurwitz-Lerch Zeta functions and their applications: Recent developments and directions for further researches. Proc. Inst. Math. Mech. Nat. Acad. Sci. Azerbaijan 2019, 45, 234-269. [CrossRef]

11. Srivastava, H.M. The Zeta and Related Functions: Recent Developments. J. Adv. Eng. Comput. 2019, 3, 329-354. [CrossRef]

12. Oldham, K.B.; Myland, J.C.; Spanier, J. An Atlas of Functions: With Equator, the Atlas Function Calculator, 2nd ed.; Springer: New York, NY, USA, 2009. 
13. Lewin, L. Polylogarithms and Associated Functions; North-Holland Publishing Co.: New York, NY, USA, 1981.

14. Weisstein, E.W. Lerch Transcendent. From MathWorld-A Wolfram Web Resource. Available online: https://mathworld.wolfram. com/LerchTranscendent.html (accessed on 1 October 2021). 